\title{
Primary Jejunal Diffuse Large B-Cell Lymphoma with Multiple Skip Ulcers and Perforation Mimicking Crohn's Disease
}

\author{
Shien-Tung Pan ${ }^{1}$, Chih-Hsin Wei ${ }^{2}$, Ming-Chi Yang ${ }^{3}$ and Shih-Sung Chuang ${ }^{*}{ }^{4}$ \\ ${ }^{1}$ Department of Pathology, Miao-Li General Hospital, Miaoli City, Taiwan; Departments of ${ }^{2}$ Hemato-oncology and \\ ${ }^{3}$ Surgery, National Taiwan University Hospital, Hsin-Chu Branch, Hsin-Chu City, Taiwan; ${ }^{4}$ Department of Pathology, \\ Chi-Mei Medical Center, Tainan and Taipei Medical University, Taipei, Taiwan
}

\begin{abstract}
Most primary intestinal lymphomas are single exophytic or annular tumor masses with diffuse large B-cell lymphoma (DLBCL) as the most common histological type. Crohn's disease is a chronic inflammatory bowel disease with multiple skip ulcers. Here we reported the case of an elderly male presented with hollow organ perforation. Segmental resection of the jejunum revealed 5 skip ulcers, grossly mimicking Crohn's disease. Pathological examination revealed diffuse sheets of large neoplastic cells expressing CD20, bcl-2, bcl-6 and MUM1 but not CD3 or CD10, a DLBCL of nongerminal center B-cell phenotype. In situ hybridization for Epstein-Barr virus was negative. Locus-specific interphase fluorescence in situ hybridization assay showed negative results for chromosomal aberrations at the IGH, $B C L 2, B C L 6$ and $M Y C$ loci. The clinical staging was IE and the patient received rituximab and combination chemotherapy. He was in complete remission for 14 months but unfortunately succumbed to an unidentified central nervous system infection 4 months later without evidence of tumor recurrence. Although multiple skip ulcers mimicked Crohn's disease, they were transverse in direction as opposed to the usual longitudinal ulcers in Crohn's disease. The correct diagnosis was reached by careful histopathological examination and ancillary studies.
\end{abstract}

Keywords: Crohn's disease, diffuse large B-cell lymphoma, intestine, primary intestinal lymphoma, Taiwan.

\section{INTRODUCTION}

The gastrointestinal tract is the most common organ for primary extranodal non-Hodgkin lymphoma (NHL) with most cases occurring as single lesions in the stomach and less commonly in the intestine [1]. Ileum and ileocecum are the most frequent locations for primary intestinal lymphomas; with diffuse large B-cell lymphoma (DLBCL) as the most common histological type [2]. The majority of primary intestinal B-cell lymphomas are exophytic or annular tumor masses, while T-cell tumors present predominantly as thickened plaques, ulcers, or strictures [3]. Crohn's disease is a chronic inflammatory disease of the intestines with fissures and ulcers, which are usually linear and longitudinal. Here we present a rare case of multicentric primary jejunal DLBCL with 5 skip ulcers mimicking Crohn's disease.

\section{CLINICAL HISTORY}

A 79-year-old man presented with severe abdominal pain for one day on December 7, 2008. Physical examination revealed peri-umbilical tenderness without lymphadenopathy. Blood routine showed leukocytosis with neutrophilia. Abdominal computed tomography (CT) revealed pneumoperitoneum, ascites and irregular-shaped thickening of the jejunal wall. Emergent operation showed dirty ascites with

*Address correspondence to this author at the Department of Pathology, ChiMei Medical Center, 901 Chung-Hwa Road, Yung-Kang, Tainan City, 710, Taiwan; Tel: 886-6-281-2811; Ext. 53686; Fax: 886-6-251-1235;

E-mail:cmh5301@mail.chimei.org.tw jejunal perforation. Segmental resection was performed, and a diagnosis of DLBCL without nodal involvement was made. He received one course of CHOP (cyclophosphamide, doxorubicin, vincristine and prednisolone) chemotherapy followed by five courses of rituximab plus CHOP. He was in complete remission for 14 months until in April 2010 when he had conscious disturbance and urinary incontinence, and CT revealed hydrocephalus without any space-occupying lesion in the brain or abdomen. He received a ventriculoperitoneal shunting procedure. Cerebrospinal fluid culture was negative and cytology revealed a mixed population of neutrophils, macrophages and small lymphocytes without lymphoma cells. He received supportive care with antibiotics and anti-fungal agents. His condition deteriorated and passed away 18 months after diagnosis.

\section{RESULTS}

Grossly, the 70-cm segment of jejunum showed 5 skip ulcers measuring up to $4 \mathrm{~cm}$ with a perforation (Fig. 1). Histopathological examination of the ulcer bases showed a diffuse lymphocytic infiltration with a focal starry-sky pattern extending to the adventitia. These lymphocytes were large with pleomorphic nuclei and prominent nucleoli without necrosis or plasmablastic differentiation. They expressed CD20, bcl-2, bcl-6 and MUM1 but not CD3, CD5, $\mathrm{CD} 10$ or CD30. The proliferation fraction was $90 \%$ by Ki- 67 immunostaining. A diagnosis of DLBCL with a non-germinal center B-cell (GCB) phenotype according to the algorithm of Hans et al. was made [4]. The tumor cells were 

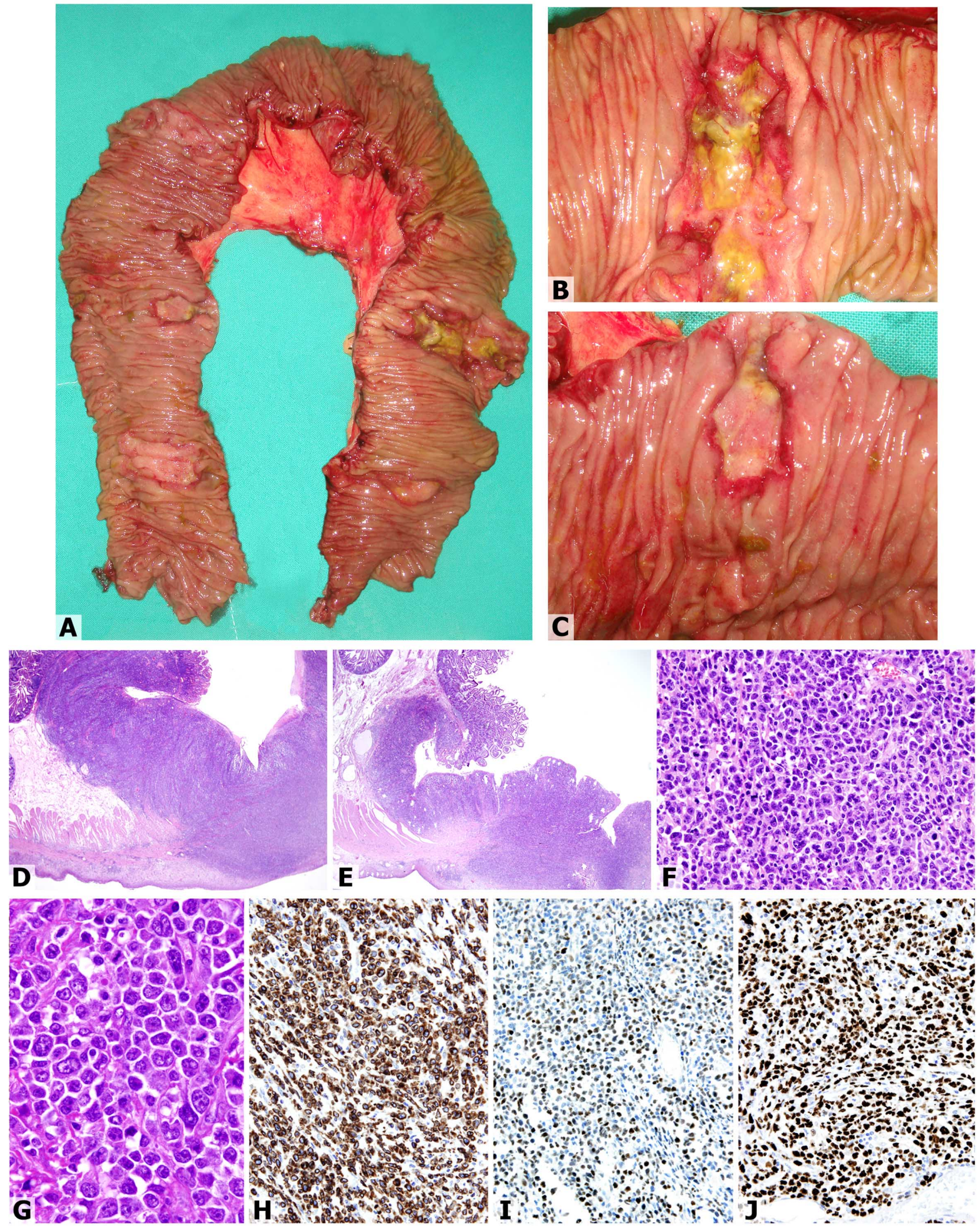

Fig. (1). A, Gross examination shows 5 skip ulcers of the small intestine with close-up views of 2 ulcers (B and C). D and E, Low-power photomicrography of 2 ulcers showing a dense and diffuse lymphocytic infiltration of the ulcer base into the adventitia (H\&E, original magnification x40). F and G. High-power and oil immersion views show large lymphoid cells with pleomorphic nuclei, frequent mitoses and abundant apoptotic bodies (H\&E, original magnification x400 and x1000, respectively). Immunohistochemically, the tumor cells express CD20, bcl-2 (H), bcl-6 (I) and MUM1 with a proliferation index of 90\% by Ki-67 (J) staining (Immunohistochemistry, original magnification, $\mathrm{x} 400)$. 
negative for Epstein-Barr virus (EBV) by in situ hybridization. Locus-specific fluorescence in situ hybridization were negative for chromosomal aberrations at the $I G H$, BCL2, BCL6 and MYC loci (Vysis, Downers Grove, IL, USA) using paraffin section with our previously described method [2].

\section{DISCUSSION}

We present an unusual primary jejunal DLBCL with multiple skip ulcers complicated by perforation. Multicentricity in intestinal lymphomas ranges from $8 \%$ to $48 \%$ in various studies, and is more frequent in T- than Bcell neoplasms $[2,3,5,6]$. Multicentricity may represent tumor progression or the specific homing property of intestinal lymphocytes. Perforation may complicate primary intestinal lymphoma and is more frequent in T- and NK/Tthan in B-cell lymphomas. The perforation rates range from 5 to $17 \%$ among patients with primary intestinal DLBCL $[2,6]$. In a previous study we have identified perforation as the single poor prognostic factor for primary intestinal DLBCLs [2]. In the current case, although there were multiple lesions and perforation, the patient responded favorably to chemotherapy but unfortunately succumbed to a central nervous system infection.

Primary intestinal lymphoma with multiple discrete ulcers may suggest Crohn's disease both clinically and pathologically. Both diseases commonly occur in the small intestine and may present with multiple ulcers or even perforation. While Crohn's disease usually involves terminal ileum and frequently extends to ileocecal valve, our case is confined to the jejunum. The gross features of Crohn's disease include multiple sharply demarcated ulcers, or aphthous ulcers, which are usually linear or serpentine and longitudinal to the long axis of the intestine. Patches or segments of normal mucosa may separate the larger ulcers, resulting in a cobblestone appearance. Involvement of submucosa may occur and may be associated with fissures or sinus tracts extending into the muscularis propria. In our case, the tumor presented with 5 discrete ulcers. Interestingly, these ulcers were transverse, in contrast to longitudinal ulcers in Crohn's disease. The distinction of lymphoma from Crohn's disease relies on the identification of atypical lymphocytes by histopathological examination and the subsequent immunohistochemical and/or other ancillary studies for confirmation. Intestinal tuberculosis was known to resemble Crohn's disease grossly as it may exhibit various combinations of mucosal ulceration, classically transverse, and inflammatory thickening of the intestinal wall, and therefore, needs to be considered in the differential diagnosis [7]. With the histological features of necrotizing granulomas and the demonstration of acid-fast bacilli, intestinal tuberculosis with ulceration can easily be differentiated from ulcerated intestinal lymphoma. Typhoid fever is a systemic infection caused by Salmonella enterica, occurring mainly in an environment with overcrowding, poor sanitation and untreated water. In rare condition $(2 \%$ of cases), typhoid fever may complicate ulcer and perforation [8]. In the fastigium stage, necrotic mucosa sloughs, producing ulcers, which hemorrhage or permeate into the peritoneal cavity. Typhoid ulcers usually have a longitudinal orientation because they are over hyperplastic and necrotic Payer patches, most commonly involving the terminal ileum.
Systemic dissemination of the organisms leads to focal granulomas, the so-called typhoid nodules, in multiple organs. These are composed of macrophages ("typhoid cells") containing ingested bacteria, erythrocytes, and degenerated lymphocytes [9]. The histology of non-caseating granulomas and evidence of bacteriology in stool culture can help making the diagnosis, which were absent in our case.

There is an increased incidence of NHL occurring in patients with inflammatory bowel diseases including Crohn's disease and ulcerative colitis. NHL occurring in patients with inflammatory bowel disease is usually extra-intestinal [10]. Rarely, primary intestinal lymphoma may occur, with bloody diarrhea as the most common presenting symptom and colorectum as the most frequent site of involvement $(60 \%)$ [11]. In our case, there was no history of chronic diarrhea to suggest inflammatory bowel disease, and furthermore, this lymphoma was in the small intestine instead of colorectum.

Although there is no standard treatment for intestinal lymphoma, chemotherapy is the mainstream modality. Franco et al. reported in their single case of primary duodenal follicular lymphoma with a 6-year complete remission after combined radio-immunotherapy [12]. The role of surgical intervention in intestinal lymphoma has been a topic of debate. Patients usually undergo surgery due to complications such as intestinal obstruction or perforation, the latter a poor prognostic factor for primary intestinal lymphoma [2,6]. Kim et al. reported in their retrospective study of 581 primary intestinal non-Hodgkin lymphomas that patients with B-cell lymphoma who underwent surgery showed a better overall survival than those did not [13]. In our patient, he achieved a complete remission for 18 months after surgery plus immunochemotherapy in spite of multiple intestinal ulcers with a perforation.

\section{CONFLICT OF INTEREST}

The authors confirm that this article content has no conflict of interest.

\section{ACKNOWLEDGEMENTS}

This work was supported by the research grand CMFHR10004 from Chi-Mei Medical Center, Tainan, Taiwan.

\section{REFERENCES}

[1] Dragosics B, Bauer P, Radaszkiewicz T. Primary gastrointestinal non-Hodgkin's lymphomas. A retrospective clinicopathologic study of 150 cases. Cancer 1985; 55: 1060-73.

[2] Chuang SS, Ye H, Yang SF, et al. Perforation predicts poor prognosis in patients with primary intestinal diffuse large B-cell lymphoma. Histopathology 2008; 53: 432-40.

[3] Domizio P, Owen RA, Shepherd NA, Talbot IC, Norton AJ. Primary lymphoma of the small intestine. A clinicopathological study of 119 cases. Am J Surg Pathol 1993; 17: 429-42.

[4] Hans CP, Weisenburger DD, Greiner TC, et al. Confirmation of the molecular classification of diffuse large B-cell lymphoma by immunohistochemistry using a tissue microarray. Blood 2004; 103: 275-82.

[5] Daum S, Ullrich R, Heise W, et al. Intestinal non-Hodgkin's lymphoma: a multicenter prospective clinical study from the German Study Group on Intestinal non-Hodgkin's Lymphoma. J Clin Oncol 2003; 21: 2740-6.

[6] Chuang SS, Chang ST, Chuang WY, et al. NK-cell lineage predicts poor survival in primary intestinal NK-cell and T-cell lymphomas. Am J Surg Pathol 2009; 33: 1230-40. 
[7] Abrams GD. Infectious Disorders of the Intestines. In: Ming SC, Goldman H, Eds. Pathology of the Gastrointestinal Tract. $2^{\text {nd }}$ ed. Baltimore: Williams \& Wilkins; 1998, pp. 662-663.

[8] Parry CM, Hien TT, Dougan G, White NJ, Farrar JJ. Typhoid fever. N Engl J Med 2002; 347: 1770-82.

[9] Genta RM, Connor DH. Infectious and parasitic diseases. In Rubin E, Farber JL Eds. Pathology, 3rd ed. Philadelphia: LippincottRaven 1999; pp. 389-92.

[10] Greenstein AJ, Mullin GE, Strauchen JA, et al. Lymphoma in inflammatory bowel disease. Cancer 1992; 69: 1119-23.
[11] Holubar SD, Dozois EJ, Loftus EV, Jr., et al. Primary intestinal lymphoma in patients with inflammatory bowel disease: A descriptive series from the prebiologic therapy era. Inflamm Bowel Dis $2010 ; 17$ : 1557-1653.

[12] Franco P, Fillip AR, Ciammella P, et al. Primary duodenal follicular lymphoma: 6-year complete remission after combined radio-immunotherapy. Acta Gastroenterol Belg 2011; 74: 337-42.

[13] Kim SJ, Choi CW, Mun YC, et al. Multicenter retrospective analysis of 581 patients with primary intestinal non-Hodgkin lymphoma from the Consortium for Improving Survival of Lymphoma (CISL). BMC Cancer 2011; 11:321-32.

Received: October 3, 2012

Revised: October 18, 2012

Accepted: October 23, 2012

(C) Pan et al.; Licensee Bentham Open.

This is an open access article licensed under the terms of the Creative Commons Attribution Non-Commercial License (http://creativecommons.org/licenses/by$\mathrm{nc} / 3.0 /$ ) which permits unrestricted, non-commercial use, distribution and reproduction in any medium, provided the work is properly cited. 